\title{
Modeling of the Process of Optimization of Decision-Making at Control of Parameters of Energy and Technical Systems on the Example of Remote Earth's Sensing Tools
}

\author{
Oleksandr Maevsky (D, Volodymyr Artemchuk (D), Yuri Brodsky (D), \\ Igor Pilkevych (D), and Pavlo Topolnitsky (D)
}

\begin{abstract}
The authors study the process of decision-making optimization in the control of the spacecraft onboard systems. To ensure the continuous operation of a remotely controlled complex technical system, it is necessary, on the basis of an analysis of the state of the onboard systems, to formulate control effects, the absence of which could lead to the system's failure to fulfill its tasks or system failure. In order to prevent such situations, an approach based on a simulation model is proposed, the use of which will reduce the risk of accidents in the onboard systems of the spacecraft. The proposed model is represented by factor space. The state of the onboard parameters of the spacecraft at different points in time is matched by the set of points that form the decision-making surface in this factor space. The basic stages of forming the optimal trajectory on the decision surface, which are approximated by numerical methods, are given and described. Using the actual values of the parameters obtained in a 15-minute data communication session from the board of the artificial satellite Earth "Ocean-1", a decision-making surface was constructed. The equation of the optimal trajectory on the created surface is obtained. The simulation results will be used to develop emergency management and control systems.
\end{abstract}

Keywords Remote earth's sensing (RES) - Spacecraft • Onboard parameters • Optimal trajectory $\cdot$ Decision surface $\cdot$ Factor space $\cdot$ Simulation model

O. Maevsky $\cdot$ Y. Brodsky $\cdot$ P. Topolnitsky

Zhytomyr National Agroecological University, Zhytomyr, Ukraine

V. Artemchuk (凶)

Pukhov Institute for Modelling in Energy Engineering of NAS of Ukraine, Kyiv, Ukraine

e-mail: ak24avo@gmail.com

I. Pilkevych

Zhytomyr Military Institute, Zhytomyr, Ukraine

(C) The Editor(s) (if applicable) and The Author(s), under exclusive license 


\section{Introduction}

In the course of its functioning, society influences the environment through wars and local conflicts, the misuse of natural resources, and accidents at industrial sites. In addition, natural disasters such as earthquakes, tsunamis, hurricanes, and more. also lead to unfavorable living conditions of mankind [1].

All this leads to the need to use environmental monitoring tools [2, 3]. For this purpose, various technical means, equipped with the equipment of registration and analysis of environmental factors are used. Today we have a tendency to increase the autonomy of these tools to improve their efficiency. In this case, there is a need for comprehensive control of the operation of these facilities to ensure their quality functioning on the one hand, and to eliminate the negative impact of these tools on the environment in the event of failure of the technical monitoring equipment. Because of their inherent benefits, one of the most commonly used environmental monitoring tools is the remote Earth's sensing (Fig. 1).

\section{Literature Analysis and Problem Statement}

In general, the task of constructing mathematical models of processes of different physical nature according to the obtained experimental data is to determine the parameters of the approximating function.

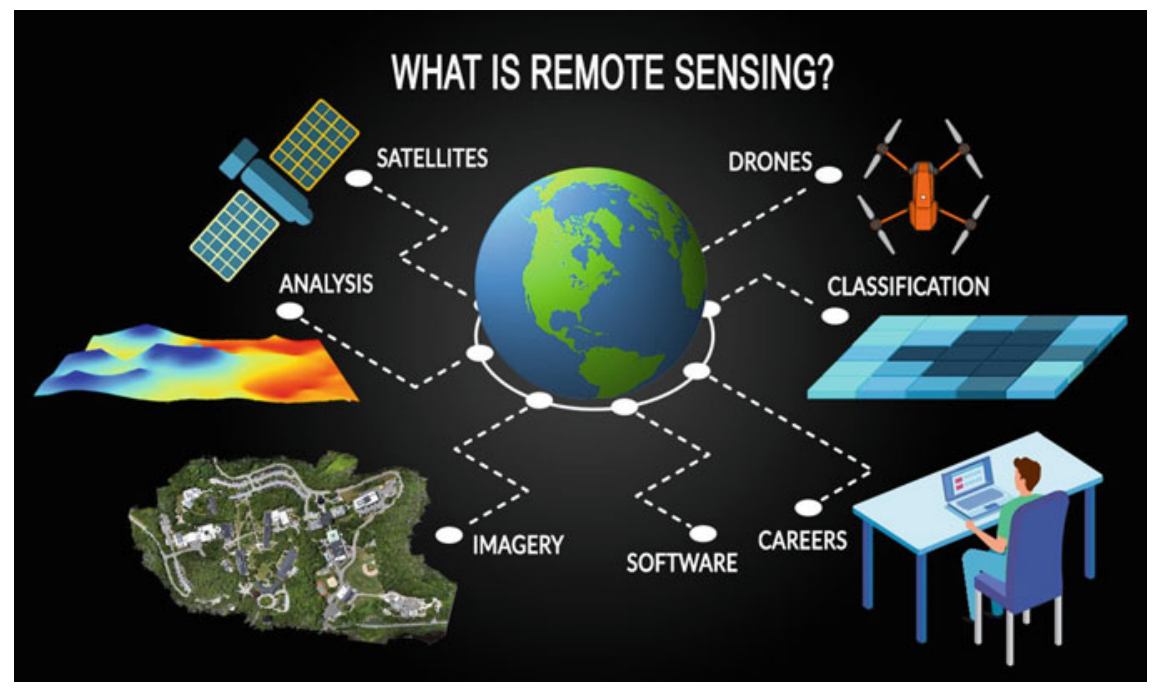

Fig. 1 Remote Earth's sensing tools [4] 
However, the rather complex nature of the dynamics of changing the parameters of the onboard systems of the means of the RES leads to complex nonlinear dependencies both in the form of approximating functions and in the form of differential equations and their systems. At the same time, the construction of an adequate mathematical model based on the experimental data provides the necessary reproduction of the dynamics of the studied process with a minimum total error and, as a consequence, increases the efficiency of control of the systems of means of RES.

When using mathematical models represented by systems of differential equations, there is a complication of computational algorithms.

For polynomial mathematical models, there is an increase in simulation errors by increasing the number of coefficients (in the corresponding functional basis), which in turn have an error in their calculation.

Methods of construction and peculiarities of creation of the mentioned mathematical models reproduced in the works of a number of scientists: S. Kuzmin, V. Baranov, E. Lviv, Yu. Linnik, V. Mudrov, V. Kushka, V. Kuntsevich, O. Kukusha, R. Kalman, E. Sage, J. Melsa, V. Medich, I. Shapiro, D. Kahaner [5-9] and others.

To prevent the occurrence of risky situations, these polynomial mathematical models and mathematical models presented by systems of differential equations, as well as stochastic mathematical models are used [10-13].

In addition, it is worth noting a number of works on optimization of decisionmaking on the management of complex technical systems [14-21].

The approach proposed in the article to create a mathematical model for preventing the occurrence of risky situations, unlike these types of models, does not require extrapolation, but is focused on the creation of the factor space of the parameters of the onboard systems of the RES means within the specified limits and the optimal control of the critical values of the control parameters of the RES systems.

\section{Purpose and Objectives of the Study}

To ensure the continuous operation of a remotely controlled complex technical system, it is necessary to formulate, on the basis of an analysis of the state of the onboard systems, control effects, the absence of which may lead to the system's failure to fulfill its tasks or system failure. In order to prevent such situations, an approach based on a simulation model is proposed, the use of which will reduce the risk of accidents in the onboard systems of the RES.

During the operation of spacecraft (SC) in the orbit of the onboard and ground control systems, the conduct and performance of a whole series of onboard mechanisms (current and voltage on the rising solar cells, heavy power plants, etc.) in the mandatory range for continuous operation of the spacecraft and exchange of information from the flight control center. These questions are used by the telemetry control system, which is created in the control of the spacecraft. The onboard SC system is used and specified throughout the work. 
However, due to various factors disclosed by the physical nature, the parameters of the onboard systems of the RES can reach critical or unacceptable values, which can be negatively observed in the mode of operation of the spacecraft, provoke the occurrence of an emergency situation and loss of spacecraft. In this case, the onboard systems accept a uniform spacecraft, always needing to optimize the operation of the onboard system and return the parameters to the range of possible meaning.

Thus, an important and relevant scientific and practical task is the efficiency of control of the onboard systems of the spacecraft. In this regard, we propose a model that optimizes the process of restoring the onboard parameters of the spacecraft within the operating range.

The proposed simulation model is a factor space. The states of the onboard parameters of the spacecraft at different times are matched by the set of points that form the decision-making surface in this factor space.

If critical or unacceptable values of the onboard parameters of the spacecraft are reached, the decision-making system of the spacecraft returns them to nominal limits, which corresponds to the movement of the end of the radius vector from point to point on the surface of decision making in factor space (Fig. 2).

To improve decision-making efficiency, it is necessary that the radius vector moves from point to point along optimal trajectories belonging to the decision surface. In this case, the optimal trajectories will be "straight" on the decision surface that has Riemann geometry. In this case, it is necessary to establish the geometry of the decision surface and find the equation of optimal trajectories on it, which is the main purpose of the conducted research.

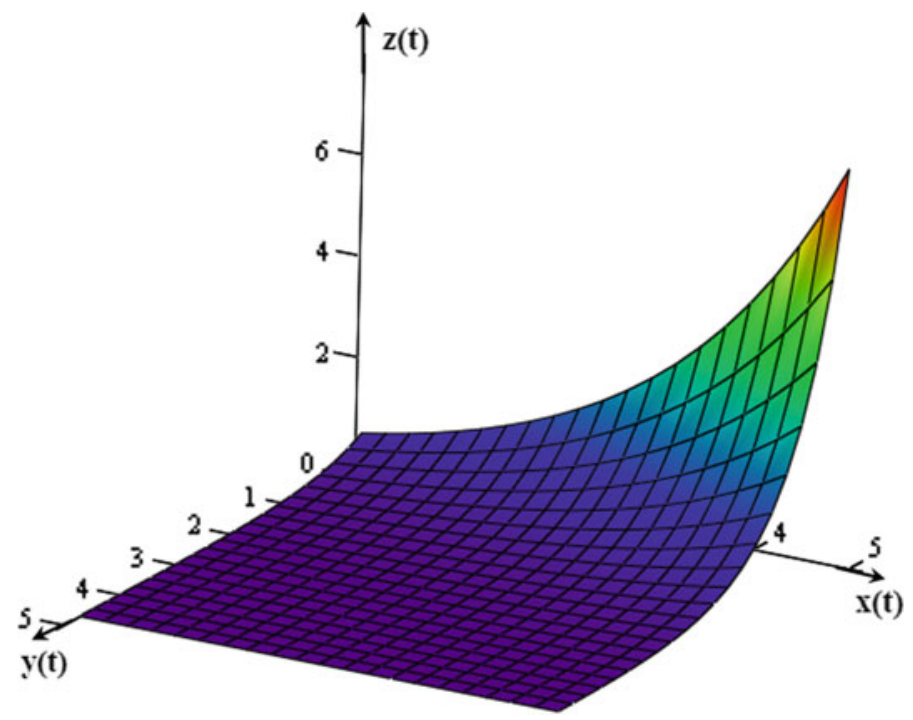

Fig. 2 Decision-making surface in factor space (units are given conventionally) 


\section{Research Methods}

This study begins with a brief analysis of publications on modeling the decisionmaking optimization process when managing the parameters of technical systems, and focuses mainly on developing a simulation model.

The following methods were used in this study: the method of comparative analysis; mathematical modeling; simulation modeling; numerical methods.

\section{Research Results}

Modeling.

As is known, the equation of optimal trajectories is obtained after equating to zero all curvilinear components of the acceleration of a point moving on a given surface.

$h_{k}=\sqrt{g_{k k}}$ - Lame coefficients, where $g_{k k}$ are the corresponding components of the metric tensor (fundamental object). Taking into account the Lame coefficients, the curvilinear components of acceleration $W_{k}$ equal to zero have the form:

$$
\sqrt{g_{k k}} \cdot W_{k}=\frac{d}{d t}\left(\frac{\partial v^{2} / 2}{\partial \ddot{q}^{k}}\right)-\frac{\partial v^{2} / 2}{\partial q^{k}}=0
$$

Given the value of the square of velocity $v^{2}=g_{i j} \dot{q}^{i} \dot{q}^{j}$, we obtain after the transformations:

$$
\ddot{q}^{m}+\frac{1}{2} g^{m k}\left[\frac{\partial g_{k j}}{\partial q^{i}}+\frac{\partial g_{i k}}{\partial q^{j}}-\frac{\partial g_{i j}}{\partial q^{k}}\right] \dot{q}^{i} \dot{q}^{j}=0
$$

where $\tilde{A}_{i j}^{m}=\frac{1}{2} g^{m k}\left[\frac{\partial g_{k j}}{\partial q^{i}}+\frac{\partial g_{i k}}{\partial q^{j}}-\frac{\partial g_{i j}}{\partial q^{k}}\right]$ is a Christoffel 2nd type affinity factor (nontensor-type).

Finally, the equation of optimal trajectories is:

$$
\ddot{q}^{m}+\tilde{A}_{i j}^{m} \dot{q}^{i} \dot{q}^{j}=0
$$

$m=1,2, \ldots, M ; M$ is the dimension of space. The system will consist of $M$ second order differential equations and have $2 M$ integration constants.

Next, we determine the Lame coefficients and analyze the applied problem for three factors.

The decision-making surface is approximated by the function of two variables of the form $z(x, y)=a_{0} a_{1}^{x} a_{2}^{y}$ (approximation implies the possibility of using other types of function). The values of the parameters $a_{0}, a_{1}, a_{2}$ are determined from the results of statistical processing. We choose scale coefficients so that the analyzed values $z, x, y$ are dimensionless. Let us introduce the generalized coordinates: 
$h$-is the height of the points of the decision surface above the $x 0 y$ plane and the azimuth angle $\varphi$ of the points of the decision surface, counterclockwise in a positive direction, that is, the generalized coordinates of the points- $(h, \varphi)$.

After no complex transformations, in generalized coordinates, the radius vector of the points on the decision surface will look like:

$$
\vec{R}(h, \phi)=\left[\begin{array}{c}
\frac{\ln \left(\frac{h}{a_{0}}\right) \operatorname{Cos}(\phi)}{\operatorname{Cos}(\phi) \ln \left(a_{1}\right)+\operatorname{Sin}(\phi) \ln \left(a_{2}\right)} \\
\frac{\ln \left(\frac{h}{a_{0}}\right) \operatorname{Sin}(\phi)}{\operatorname{Cos}(\phi) \ln \left(a_{1}\right)+\operatorname{Sin}(\phi) \ln \left(a_{2}\right)} \\
h
\end{array}\right]=\left[\begin{array}{c}
\frac{\ln \left(\frac{h}{a_{0}}\right)}{\ln \left(a_{1}\right)+\operatorname{tg}(\phi) \ln \left(a_{2}\right)} \\
\frac{\ln \left(\frac{h}{a_{0}}\right)}{\operatorname{ctg}(\phi) \ln \left(a_{1}\right)+\ln \left(a_{2}\right)} \\
h
\end{array}\right]
$$

We introduce the notation $\frac{1}{a_{0}}=A_{0}, \ln \left(a_{1}\right)=A_{1}, \ln \left(a_{2}\right)=A_{2}$. Determine the local basis (benchmark):

$$
\vec{g}_{\phi}=\left[\begin{array}{c}
-\frac{A_{2} \ln \left(A_{0} h\right)}{\left(A_{1} \operatorname{Cos}(\phi)+A_{2} \operatorname{Sin}(\phi)\right)^{2}} \\
\frac{A_{1} \ln \left(A_{0} h\right)}{\left(A_{1} \operatorname{Cos}(\phi)+A_{2} \operatorname{Sin}(\phi)\right)^{2}} \\
0
\end{array}\right] ; \quad \vec{g}_{h}=\left[\begin{array}{c}
\frac{1}{\left(A_{1}+A_{2} \operatorname{tg}(\phi)\right) h} \\
\frac{1}{\left(A_{2}+A_{1} \operatorname{ctg}(\phi)\right) h} \\
1
\end{array}\right]
$$

We introduce additional notations to simplify the calculations:

$$
\begin{aligned}
& B(\phi)=A_{2} \operatorname{Sin}(\phi)+A_{1} \operatorname{Cos}(\phi) ; \\
& M(\phi)=A_{1}+A_{2} \operatorname{tg}(\phi) ; \\
& N(\phi)=A_{2}+A_{1} \operatorname{ctg}(\phi) ;
\end{aligned}
$$

Given the notation (6), the metric tensor will have the form:

$$
g_{i j}=\left[\begin{array}{cc}
\left(\frac{\ln \left(A_{0} h\right)}{B^{2}(\phi)}\right)^{2}\left(A_{1}^{2}+A_{2}^{2}\right) & \frac{\ln \left(A_{0} h\right)}{B^{2}(\phi) h}\left(\frac{A_{1}}{N(\phi)}-\frac{A_{2}}{M(\phi)}\right) \\
\frac{\ln \left(A_{0} h\right)}{B^{2}(\phi) h}\left(\frac{A_{1}}{N(\phi)}-\frac{A_{2}}{M(\phi)}\right) & \frac{1}{h^{2}}\left(\frac{1}{M^{2}(\phi)}+\frac{1}{N^{2}(\phi)}\right)+1
\end{array}\right]
$$

To calculate the values of the symbols of the 2 nd type of Christoffel, we find the determinant $g$ of the metric tensor $g_{i j}$ and the object of the upper structure $g^{p i}$ :

$$
\begin{aligned}
g= & \left(\frac{\ln \left(A_{0} h\right)}{B^{2}(\phi)}\right)^{2}\left(A_{1}^{2}+A_{2}^{2}\right) \\
& \times\left(\frac{1}{h^{2}}\left(\frac{1}{M^{2}(\phi)}+\frac{1}{N^{2}(\phi)}\right)+1\right)-\left(\frac{\ln \left(A_{0} h\right)}{B^{2}(\phi) h}\left(\frac{A_{1}}{N(\phi)}-\frac{A_{2}}{M(\phi)}\right)\right)^{2}
\end{aligned}
$$




$$
g^{p i}=\left[\begin{array}{ll}
\left(\frac{1}{h^{2}}\left(\frac{1}{M^{2}(\phi)}+\frac{1}{N^{2}(\phi)}\right)+1\right) / g & \left(-\frac{\ln \left(A_{0} h\right)}{B^{2}(\phi) h}\left(\frac{A_{1}}{N(\phi)}-\frac{A_{2}}{M(\phi)}\right)\right) / g \\
\left(-\frac{\ln \left(A_{0} h\right)}{B^{2}(\phi) h}\left(\frac{A_{1}}{N(\phi)}-\frac{A_{2}}{M(\phi)}\right)\right) / g & \left(\frac{\ln \left(A_{0} h\right)}{B^{2}(\phi)}\right)^{2}\left(A_{1}^{2}+A_{2}^{2}\right) / g
\end{array}\right]
$$

Enter the designation of generalized coordinates:

$$
q^{1}=\phi, q^{2}=h
$$

To reconcile the index notation, we rewrite (3) in the following representation:

$$
\ddot{q}^{p}+\tilde{A}_{k l}^{p} \dot{q}^{k} \dot{q}^{l}=0
$$

Symbol of the 2nd type of Christoffel:

$$
\tilde{A}_{k l}^{p}=\frac{1}{2} g^{p i}\left[\frac{\partial g_{i k}}{\partial q^{l}}+\frac{\partial g_{i l}}{\partial q^{k}}-\frac{\partial g_{k l}}{\partial q^{i}}\right]
$$

In expanded form it will look like:

$$
\tilde{A}_{k l}^{p}=\left(\begin{array}{cc}
\tilde{A}_{11}^{1} & \tilde{A}_{21}^{1} \\
\tilde{A}_{12}^{1} & \tilde{A}_{22}^{1} \\
\tilde{A}_{11}^{2} & \tilde{A}_{21}^{2} \\
\tilde{A}_{12}^{2} & \tilde{A}_{22}^{2}
\end{array}\right)
$$

\subsection{Interpretation of Results and Their Evaluation}

To construct the decision-making surface, we will use the actual values of the parameters obtained in a 15-minute session of data transmission from the board of an artificial satellite of the Earth "Ocean-1". The parameters studied have a sufficient level of correlation. The first parameter under study is the current of solar cells, denote it as $z(t)$, the second parameter under test is the load current, denote it as $x(t)$, the third parameter under test is the load voltage, denote it as $y(t)$. All actual parameters for a session are listed in Table 1.

Using numerical methods we obtain an approximate equation of the decision surface:

$$
z(x, y)=0,00645 \cdot(23,976)^{x} \cdot(17,644)^{y}
$$

A fragment of the matrix ( $\operatorname{size} 30 \times 30$ ) with relative values $z(x, y)=S$ is shown in the Fig. 3: 
Table 1 The values of the investigated parameters per communication session

\begin{tabular}{|c|c|c|c|c|c|c|}
\hline \multirow[t]{3}{*}{ Session time, min } & \multicolumn{2}{|c|}{$\begin{array}{l}\text { Current of solar panels } \\
\text { (TC-1), z(t), A }\end{array}$} & \multicolumn{2}{|c|}{$\begin{array}{l}\text { Load current }(\mathrm{TH}-1) \\
\mathrm{x}(\mathrm{t}), \mathrm{A}\end{array}$} & \multicolumn{2}{|c|}{$\begin{array}{l}\text { Load voltage }(\mathrm{HH}-1) \\
\mathrm{y}(\mathrm{t}), \mathrm{V}\end{array}$} \\
\hline & \multicolumn{2}{|c|}{ Range $\left(A_{\max }=21 A\right)$} & \multicolumn{2}{|c|}{ Range $\left(A_{\max }=50 A\right)$} & \multicolumn{2}{|c|}{ Range $\left(\mathrm{U}_{\max }=34 \mathrm{~V}\right)$} \\
\hline & A & $\% / 100$ & A & $\% / 100$ & $\mathrm{U}$ & $\% / 100$ \\
\hline 0 & 6.69 & 0.318571 & 5.77 & 0.1154 & 32.12 & 0.944706 \\
\hline 0.5 & 6.09 & 0.29 & 10.08 & 0.2016 & 31.8 & 0.935294 \\
\hline 1 & 1.28 & 0.060952 & 5.12 & 0.1024 & 31.59 & 0.929118 \\
\hline 1.5 & 0.57 & 0.027143 & 6.56 & 0.1312 & 33 & 0.970703 \\
\hline 2 & 4.24 & 0.201987 & 9.93 & 0.198586 & 31.59 & 0.929171 \\
\hline 2.5 & 1.86 & 0.088792 & 8.87 & 0.177369 & 32.2 & 0.947124 \\
\hline 3 & 5.11 & 0.243274 & 11.25 & 0.2251 & 32.18 & 0.946418 \\
\hline 3.5 & 2.37 & 0.112661 & 14.7 & 0.293948 & 31.22 & 0.918111 \\
\hline 4 & 1.63 & 0.077577 & 10.13 & 0.202648 & 32.79 & 0.964451 \\
\hline 4.5 & 3.04 & 0.14472 & 11.53 & 0.230511 & 31.58 & 0.928816 \\
\hline 5 & 2 & 0.095192 & 10.42 & 0.208421 & 32.36 & 0.951876 \\
\hline 5.5 & 2.99 & 0.142477 & 12.64 & 0.25275 & 30.59 & 0.899635 \\
\hline 6 & 3.4 & 0.162056 & 10.7 & 0.213901 & 32.06 & 0.943068 \\
\hline 6.5 & 4.29 & 0.20443 & 12.43 & 0.248514 & 32.31 & 0.950285 \\
\hline 7 & 2.95 & 0.140634 & 4.76 & 0.095147 & 32.85 & 0.966163 \\
\hline 7.5 & 5.8 & 0.276256 & 13.42 & 0.268416 & 30.25 & 0.88965 \\
\hline 8 & 1.63 & 0.077591 & 7.94 & 0.158792 & 32.17 & 0.946271 \\
\hline 8.5 & 4.94 & 0.235122 & 4.17 & 0.083367 & 30.39 & 0.893866 \\
\hline 9 & 5.89 & 0.280563 & 13.38 & 0.267676 & 33.18 & 0.975891 \\
\hline 9.5 & 6.25 & 0.297733 & 11.32 & 0.226454 & 31.8 & 0.935411 \\
\hline 10 & 2.83 & 0.134952 & 6.31 & 0.126161 & 30.35 & 0.892605 \\
\hline 10.5 & 3.76 & 0.178893 & 6.97 & 0.139363 & 32.59 & 0.958582 \\
\hline 11 & 3.96 & 0.188503 & 6.63 & 0.132647 & 32.4 & 0.953046 \\
\hline 11.5 & 4.68 & 0.222879 & 6.04 & 0.120858 & 30.42 & 0.894815 \\
\hline 12 & 3.27 & 0.155753 & 12.38 & 0.247658 & 30.37 & 0.893201 \\
\hline 12.5 & 4.23 & 0.201609 & 13.67 & 0.273471 & 32.33 & 0.950742 \\
\hline 13 & 4.43 & 0.210772 & 8.38 & 0.16762 & 32.66 & 0.960679 \\
\hline 13.5 & 6.39 & 0.304152 & 12.83 & 0.256608 & 32.21 & 0.947246 \\
\hline 14 & 6.61 & 0.314822 & 12.05 & 0.240982 & 32.76 & 0.963529 \\
\hline 14.5 & 2.29 & 0.108869 & 8.09 & 0.161817 & 30.89 & 0.908413 \\
\hline 15 & 4.22 & 0.200917 & 7.07 & 0.141479 & 31.66 & 0.931191 \\
\hline
\end{tabular}




\begin{tabular}{|r|r|r|r|r|r|r|r|r|r|}
\hline & \multicolumn{1}{|c|}{0} & \multicolumn{1}{c|}{1} & \multicolumn{1}{c|}{2} & \multicolumn{1}{c|}{3} & \multicolumn{1}{c|}{4} & \multicolumn{1}{c|}{5} & \multicolumn{1}{c|}{6} & \multicolumn{1}{c|}{7} & \multicolumn{1}{c|}{8} \\
\hline 0 & 0.118 & 0.128 & 0.106 & 0.114 & 0.125 & 0.134 & 0.121 & 0.13 & 0.12 \\
\hline 1 & 0.135 & 0.146 & 0.122 & 0.131 & 0.143 & 0.153 & 0.139 & 0.149 & 0.138 \\
\hline 2 & 0.177 & 0.193 & 0.16 & 0.172 & 0.188 & 0.202 & 0.183 & 0.196 & 0.181 \\
\hline 3 & 0.15 & 0.164 & 0.136 & 0.146 & 0.16 & 0.171 & 0.155 & 0.167 & 0.154 \\
\hline 4 & 0.21 & 0.228 & 0.189 & 0.204 & 0.222 & 0.238 & 0.216 & 0.232 & 0.214 \\
\hline 5 & 0.133 & 0.145 & 0.12 & 0.129 & 0.141 & 0.151 & 0.137 & 0.147 & 0.136 \\
\hline 6 & 0.194 & 0.211 & 0.175 & 0.188 & 0.205 & 0.22 & 0.2 & 0.215 & 0.198 \\
\hline 7 & 0.146 & 0.158 & 0.131 & 0.142 & 0.154 & 0.166 & 0.15 & 0.161 & 0.149 \\
\hline 8 & 0.125 & 0.136 & 0.113 & 0.122 & 0.133 & 0.143 & 0.129 & 0.139 & 0.128 \\
\hline 9 & 0.13 & 0.142 & 0.118 & 0.127 & 0.138 & 0.148 & 0.134 & 0.144 & 0.133 \\
\hline 10 & 0.235 & 0.256 & 0.213 & 0.229 & 0.25 & 0.268 & 0.243 & 0.261 & 0.241 \\
\hline 11 & 0.128 & 0.139 & 0.115 & 0.124 & 0.136 & 0.145 & 0.132 & 0.142 & 0.131 \\
\hline 12 & 0.118 & 0.129 & 0.107 & 0.115 & 0.126 & 0.135 & 0.122 & 0.131 & 0.121 \\
\hline 13 & 0.171 & 0.186 & 0.154 & 0.166 & 0.181 & 0.194 & 0.176 & 0.189 & 0.175 \\
\hline 14 & 0.179 & 0.195 & 0.162 & 0.175 & 0.19 & 0.204 & 0.185 & 0.199 & 0.184 \\
\hline 15 & 0.132 & 0.144 & 0.119 & 0.129 & 0.14 & 0.15 & 0.136 & 0.146 & $\ldots$ \\
\hline
\end{tabular}

Fig. 3 A fragment of the matrix (size $30 \times 30$ ) with relative values $z(x, y)=S$

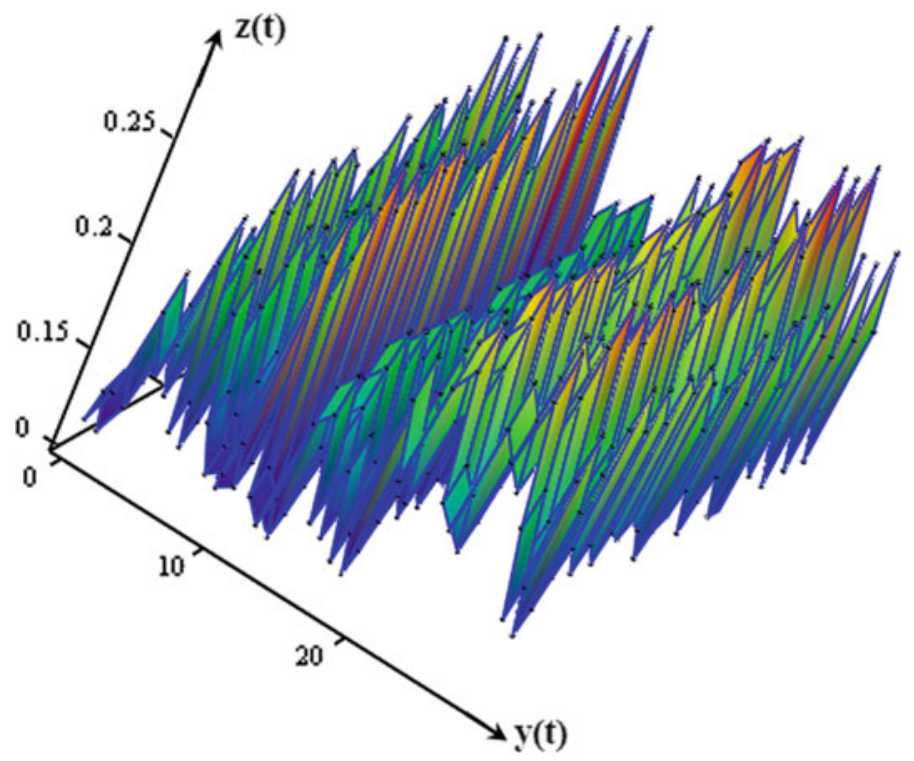

Fig. 4 Approximation of the decision surface $z(x, y)=S$ 


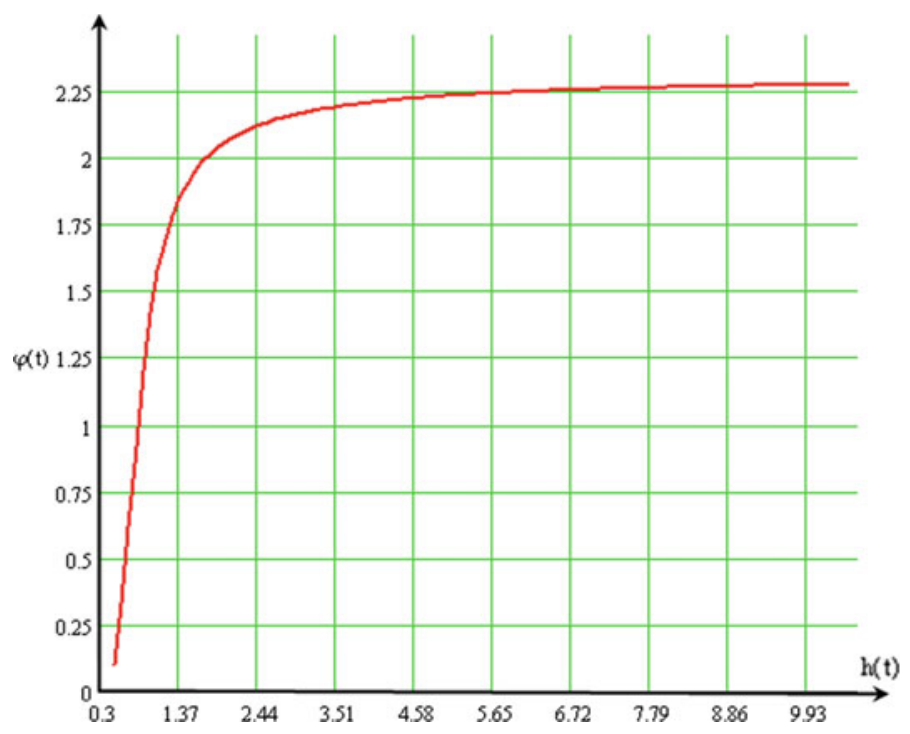

Fig. 5 The equation of the optimal trajectory on the decision surface $z(x, y)=S$

Figure 4 presents an approximation of the decision-making surface based on the data of the matrix of relative quantities $z(x, y)=S$.

Let us calculate the symbols of the 2 nd type of Christoffel $\tilde{A}_{11}^{1}$ and $\tilde{A}_{21}^{2}$ using Mathcad and obtain the following system of differential equations:

$$
\left\{\begin{array}{c}
\ddot{q}^{1}+\tilde{A}_{11}^{1}\left(\dot{q}^{1}\right)^{(2)}=0 \\
\ddot{q}^{2}+\tilde{A}_{21}^{2} \dot{q}^{2} \dot{q}^{1}=0
\end{array}\right.
$$

which allowed us to construct the required equation $\varphi(h)$ presented in Fig. 5, respectively. Substituting into the system (11) the other values of the Christoffel 2nd type symbols, we obtain variants of optimal trajectories on the decision surface.

\section{Discussion of the Results}

Further development of the model involves the study of the influence of errors arising from the approximation of the actual decision surface on the process of optimal control of given parameters. As well as adapting the model to the factor space of higher dimensions. 


\section{Conclusions}

The proposed model allows optimal control of the set parameters of technical systems in order to prevent emergencies and risk situations. Based on this model, it is possible to create decision support systems for organizing activities aimed at reducing risk situations in technical systems.

\section{References}

1. Popov, O., Iatsyshyn, A., Kovach, V., Artemchuk, V., Taraduda, D., Sobyna, V., Sokolov, D., Dement, M., Yatsyshyn, T., Matvieieva, I.: Analysis of possible causes of npp emergencies to minimize risk of their occurrence. Nucl. Radiati. Saf. 1(81), 75-80 (2019). https://doi.org/10. 32918/nrs.2019.1(81).13

2. Popov, O.O., Iatsyshyn, A.V., Kovach, V.O., Artemchuk, V.O., Kameneva, I.P., Taraduda, D.V., Sobyna, V.O., Sokolov, D.L., Dement, M.O., Yatsyshyn, T.M.: Risk assessment for the population of Kyiv, Ukraine as a result of atmospheric air pollution. J. Health Poll. 10(25), 200303 (2020). https://doi.org/10.5696/2156-9614-10.25.200303

3. Popov, O., Iatsyshyn, A., Kovach, V., Artemchuk, V., Taraduda, D., Sobyna, V., Sokolov, D., Dement, M., Hurkovskyi, V., Nikolaiev, K., Yatsyshyn, T., Dimitriieva, D.: Physical features of pollutants spread in the air during the emergency at NPPs. Nucl. Radiat. Saf. 4(84), 88-98 (2019). https://doi.org/10.32918/nrs.2019.4(84).11

4. What is Remote Sensing? The Definitive Guide. Access mode: https://gisgeography.com/rem ote-sensing-earth-observation-guide/

5. Shmelova, T., Sikirda, Y., Scarponi, C., Chialastri, A.: Deterministic and stochastic models of decision making in air navigation socio-technical system. In: CEUR Workshop Proceedings, vol. 2104, pp. 649-656 (2018). http://ceur-ws.org/Vol-2104/paper_221.pdf

6. Grishin, I., Timirgaleeva, R.: Air navigation: automation method for controlling the process of detecting aircraft by a radar complex. In 2019 24th Conference of Open Innovations Association, pp. 110-115 (2019). https://doi.org/10.23919/fruct.2019.8711905

7. Kondratyeva, N., Valeev, S.: Fatigue test optimization for complex technical system on the basis of lifecycle modeling and big data concept. In 2016 IEEE 10th International Conference on Application of Information and Communication Technologies, pp. 1-4 (2016). https://doi. org/10.1109/icaict.2016.7991656

8. Leveson, N.: A new accident model for engineering safer systems. Saf. Sci. 42(4), 237-270 (2004)

9. Raza, A.: Mathematical model of corrective maintenance based on operability checks for safety critical systems. Am. J. Applied Math. 6(1), 8-14 (2018)

10. Mokhor, V., Gonchar, S., Dybach, O.: Methods for the total risk assessment of cybersecurity of critical infrastructure facilities. Nucl. Radiat. Saf. 2(82), 4-8 (2019). https://doi.org/10.32918/ nrs.2019.2(82).01

11. Bilan, T., Rezvik, I., Sakhno, O., But, O., Bogdanov, S.: Main approaches to cable aging management at nuclear power plants in Ukraine. Nucl. Radiat. Saf. 4(84), 54-62 (2019). https:// doi.org/10.32918/nrs.2019.4(84).07

12. Mokhor, V.V., Tsurkan, O.V., Tsurkan, V.V., Herasymov, R.P.: Information security assessment of computer systems by socio-engineering approach. In: Selected Papers of the XVII International Scientific and Practical Conference on Information Technologies and Security (ITS 2017), November 30, 2017, pp. 92-98. Kyiv, Ukraine (2017). http://ceur-ws.org/Vol-2067/pap er13.pdf 
13. Yatsyshyn, T., Shkitsa, L., Popov, O., Liakh, M.: Development of mathematical models of gas leakage and its propagation in atmospheric air at an emergency gas well gushing. East. Eur. J. Enter. Technol. 5/10(101), 49-59 (2019). https://doi.org/10.15587/1729-4061.2019.179097

14. Zhang, S., Du, M., Tong, J., Li, Y.-F.: Multi-objective optimization of maintenance program in multi-unit nuclear power plant sites. Reliab. Eng. Sys. Saf. (2019). https://doi.org/10.1016/ j.ress.2019.03.034

15. Chen, Z.C., Liu, P.H., Pei, Z.: An approach to multiple attribute group decision making based on linguistic intuitionistic fuzzy numbers. Int. J. Comput. Int. Sys. 8, 747-760 (2015)

16. Tang, J., Meng, F., Zhang, Y.: Decision making with interval-valued intuitionistic fuzzy preference relations based on additive consistency analysis. Inf. Sci. 467, 115-134 (2018). https:// doi.org/10.1016/j.ins.2018.07.036

17. Kovach, V., Lysychenko, G. Toxic soil contamination and its mitigation in Ukraine. In: Dent, D., Dmytruk, Y. (eds.) Soil Science Working for a Living. Springer, Cham (2017). https://doi. org/10.1007/978-3-319-45417-7_18

18. Gomez Fernandez, M., Tokuhiro, A., Welter, K., Wu, Q.: Nuclear energy system's behavior and decision making using machine learning. Nucl. Eng. Des. 324, 27-34 (2017). https://doi. org/10.1016/j.nucengdes.2017.08.020

19. Reyes, J.N., Groome, J., Woods, B.G., Young, E., Abel, K., Yao, Y., Yoo, Y.J.: Testing of the multi-application small light water reactor (MASLWR) passive safety systems. Nucl. Eng. Des. 237(18), 1999-2005

20. Popov, O., Iatsyshyn, A., Kovach, V., Artemchuk, V., Taraduda, D., Sobyna, V., Sokolov, D., Dement, M., Yatsyshyn, T.: Conceptual approaches for development of informational and analytical expert system for assessing the NPP impact on the environment. Nucl. Radiat. Saf. 3(79), 56-65 (2018). https://doi.org/10.32918/nrs.2018.3(79).09

21. Wang, D., Gu, X., Zhou, G., Li, S., Liang, H.: Decision-making optimization of power system extended black-start coordinating unit restoration with load restoration. Int. Trans. Elec. Energy Sys. 27(9), e2367 (2007). https://doi.org/10.1002/etep.2367 